\title{
miR-363 acts as a tumor suppressor in osteosarcoma cells by inhibiting PDZD2
}

\author{
FAN HE ${ }^{1}$, LONG FANG $^{2}$ and QINGSHUI YIN ${ }^{1}$ \\ ${ }^{1}$ Department of Orthopedic, The First School of Clinical Medicine, Southern Medical University, \\ Guangzhou, Guangdong 510515; ${ }^{2}$ Department of Orthopedic, Shandong Academy of Medical Sciences, \\ Shandong Cancer Hospital Affiliated to Shandong University, Jinan, Shandong 250117, P.R. China
}

Received October 11, 2018; Accepted March 13, 2019

DOI: $10.3892 / o r .2019 .7078$

\begin{abstract}
PDZ domain containing 2 (PDZD2) is a multi-PDZ domain protein that promotes the proliferation of insulinoma cells, and is upregulated during prostate tumorigenesis. However, the function of PDZD2 in other cancers, including osteosarcoma (OS), remains unclear. Dysregulation of microRNAs (miRNAs) contributes to tumor initiation, proliferation and metastasis, via the regulation of their target genes. The present study investigated the functions of miR-363 and PDZD2 in MG-63 OS cells. The results revealed that MG-63 cells contained low levels of miR-363, and that overexpression of miR-363 in MG-63 cells significantly inhibited the vitality, proliferation, and colony formation ability of the cells, but promoted their apoptosis and G1/S arrest by regulating proliferating cell nuclear antigen (PCNA) and caspase-3 expression. Additionally, miR-363 impaired the migration and invasion of MG-63 cells by regulating the epithelial-mesenchymal transition (EMT) phenotype. Notably, a bioinformatics analysis and luciferase reporter assay indicated that PDZD2 was a direct target of miR-363. miR-363 overexpression reduced PDZD2 protein levels and knockdown of PDZD2 suppressed the colony formation, migration and invasion of MG-63 cells, but promoted their apoptosis by regulating expression of PCNA, caspase-3, and the EMT phenotype. In vivo studies further confirmed that miR-363 functioned as tumor suppressor, by inhibiting tumor growth, promoting cell apoptosis, and reducing PDZD2 and PCNA levels and the prevalence of the EMT phenotype in tumor tissues. The present data demonstrated that downregulation of the tumor suppressor miR-363 may be involved in the development of osteosarcoma via regulation of PDZD2.
\end{abstract}

Correspondence to: Dr Qingshui Yin, Department of Orthopedic, The First School of Clinical Medicine, Southern Medical University, 111 Liuhua Road, Guangzhou, Guangdong 510515, P.R. China E-mail: yqs8521@163.com

Key words: miR-363, PDZ domain containing 2, osteosarcoma, apoptosis, epithelial-mesenchymal transition

\section{Introduction}

As the most common primary malignant bone tumor, osteosarcoma (OS) is a leading cause of cancer death among children and adolescents, and accounts for $\sim 60 \%$ of all malignant bone tumors (1). The many advances made in treating OS (for example, the use of surgery combined with neoadjuvant and adjuvant chemotherapy) have significantly improved patient outcomes, and $60-70 \%$ of OS patients now survive their disease. However, several patients, who are resistant to available chemotherapeutics or with metastatic disease at diagnosis, have far worse clinical outcomes, and their survival rate is $<30 \%(2-4)$.

PSD95/Discs-large/ZO-1 (PDZ) domain containing 2 (PDZD2), also referred to as KIAA0300, PIN-1, PAPIN, activated in prostate cancer (AIPC) and PDZ domain-containing protein 3 (PDZK3), is a six-PDZ domain protein (5). PDZD2 is ubiquitously expressed in multiple tissues, including heart, brain, liver, spleen, lung, and kidney, but is expressed at exceptionally high levels in the pancreas and certain cancer tissues, such as prostate cancer (6-8). An early study reported that the PDZD2 transcript is upregulated in human prostate tumor cells and the AIPC protein is abundantly expressed in high-grade premalignant lesions of the prostate and in human prostate tumors, suggesting that activation of PDZD2 expression may be an early event in human prostate tumorigenesis (7). During a recent evaluation of single nucleotide polymorphisms (SNPs) associated with the risk for renal cell carcinoma (RCC), Zhang et al (9) demonstrated that Rs10054504 (5p13.3), which is located in intron 4 of PDZD2, was significantly associated with the risk for RCC in a Chinese population. However, the role of PDZD2 in osteosarcoma remains unclear.

The vast majority of RNA transcripts in mammalian cells originate from genes that do not code for proteins, and are processed to generate different classes of RNAs with different sizes (10). The most investigated type of such RNAs are microRNAs (miRNAs), which are small non-coding RNA molecules of 18-22 nucleotides in length that regulate gene expression at the post-transcriptional level by interacting with complementary sequences in the 3'-UTRs of their target mRNAs to inhibit their expression (11). Aberrant miRNA expression has been recognized as a critical event during carcinogenesis, and depending on the tumor type, may serve 
either to inhibit or enhance tumor growth. For example, miR-7, miR-15/16, miR-124, and miR-363 have been demonstrated to suppress tumor growth, while miR-155, miR-9, miR-708, and miR-224 can function as oncogenes (12-14). Tian et al (15) reported that miR-15a expression is downregulated in osteosarcoma tissues. miR-15a serves to inhibit cell proliferation, migration, and invasion by targeting the $\mathrm{TNF} \alpha-$ induced protein 1 gene. Decreased levels of miR-382, which targets Kruppel-like factor 12 and homeodomain interacting protein kinase 3 , were reported in tumor specimens from OS patients with poor response to chemotherapy, compared with specimens obtained from patients with good response to chemotherapy (16). miR-363 has exhibited tumor suppressive effects in numerous types of cancer, including colorectal cancer (17), hepatocellular carcinoma (18), gallbladder cancer (19) and breast cancer (20). However, the tumor suppressive function of miR-363 in OS requires further investigation.

In the present study, a bioinformatics analysis was performed and the results identified the PDZD2 gene as a direct target of miR-363 in OS. Restoration of miR-363 expression and knockdown of PDZD2 impaired the typical characteristics of OS tumor cells, including their proliferation, evasion of apoptosis, and metastasis.

\section{Materials and methods}

Cell lines and reagents. Three OS cell lines (MG-63, HOS, and Saos2) and one normal human osteoblastic cell line (hFOB1.19) were used in the present study. These cell lines were purchased from the cell bank of the Type Culture Collection of the Chinese Academy of Sciences (Shanghai, China). The OS cell lines were cultured in Dulbecco's modified Eagle's medium (DMEM) supplemented with $10 \%$ fetal bovine serum (FBS; Thermo Fisher Scientific, Inc., Waltham, MA, USA), ampicillin, and streptomycin at $37^{\circ} \mathrm{C}$ with $5 \% \mathrm{CO}_{2}$. The hFOB 1.19 cells were routinely maintained in DMEM/ Ham's F12 medium (DMEM/F12; 1:1 w/w mix) containing $10 \%$ FBS and $300 \mu \mathrm{g} / \mathrm{ml}$ neomycin (G418) at $34 \mathrm{oC}$ with $5 \%$ $\mathrm{CO} 2$. Antibodies targeting GAPDH, E-cadherin, PDZD2, proliferating cell nuclear antigen (PCNA), cleaved caspase-3 and vimentin were obtained from Cell Signaling Technology, Inc. (Danvers, MA, USA) and Abcam (Cambridge, MA, USA). The miR-363 mimics (5'-AAUUGCACGGUAUCCAUCU GUA-3') and negative control (5'-UUCUCCGAACGUGU CACGUTT-3') oligonucleotides were purchased from GenePharma Co., Ltd. (Shanghai, China). Small interfering RNA (siRNA) targeting PDZD2 (siRNA-PDZD2) (139, 5'-GCUGAACUUUGCUGUGGAUUU-3'; 580, 5' -CUCUG AACCAGGAGAAACAUU-3'; and 1027, 5'-GCUGGGAAU UCAGGUUAGUUU-3'), pcDNA 3.1-NEAT1, and the negative controls were prepared by RiboBio Co., Ltd. (Guangzhou, China). The psiCHECK2-UTR (wild-type and mutant) of PDZD2 was prepared by GenePharma Co., Ltd.

RNA isolation and reverse transcription-quantitative polymerase chain reaction (RT- $q P C R)$. RT-qPCR was performed to determine the expression of miR-363 and PDZD2 in tumor cells. Total RNA was extracted from tissues or cell lines using TRIzol reagent (Thermo Fisher Scientific, Inc.) according to a standard RNA isolation protocol. Briefly, for the detection of mature miR-363, poly-A polymerase was used to attach poly-A tails onto the 3 ' end of miR-363 molecules, and High-capacity cDNA Reverse Transcriptase kit (Applied Biosystems; Thermo Fisher Scientific, Inc.) was used to reverse transcribe the polyA-tailed miRNAs by use of a unique oligo (dT) adaptor primer, according to the manufacturer's protocol (Takara Biotechnology Co., Ltd., Dalian, China). The reaction conditions used for qPCR were as follows: incubation at $95^{\circ} \mathrm{C}$ for $60 \mathrm{sec}$, followed by 40 cycles of $95^{\circ} \mathrm{C}$ for $5 \mathrm{sec}$, and $60^{\circ} \mathrm{C}$ for 34 sec. miR-363 and PDZD2 levels were quantified by using SYBR Premix Ex Taq (Takara Biotechnology Co., Ltd.) on an ABI 7500 Fast Sequence Detection System (Applied Biosystems; Thermo Fisher Scientific, Inc.). The levels of miR-363 and PDZD2 were normalized to those of U6 and GAPDH, respectively. The primers were: miR-363, forward 5'-ACACTCCAGCTGGGAATTGCACGGTATCCATC-3' and reverse 5'-CTCAACTGGTGTCGTGGAGTCGGCA ATTCAGTTGAGTACAGATG-3'; U6, forward 5'-CTCG CTTCGGCAGCACA-3' and reverse 5'-AACGCTTCACG AATTTGCGT-3'; PDZD2, forward 5'-TCTGTACTG TGTACCTCACCAA-3' and reverse 5'-CCCTGCGCTTTTC ACCATAG-3'; and GAPDH, forward 5'-TGTTCGTCATG GGTGTGAAC-3' and reverse 5'-ATGGCATGGACTG TGGTCAT-3'. Relative fold changes in mRNA expression were calculated using the formula $2^{-\Delta \Delta C q}(21)$.

Western blot analysis. Total proteins in tissues or cell lines were extracted with RIPA cell lysis buffer (Santa Cruz Biotechnology, Inc., Dallas, TX, USA), and the concentrations of these proteins were determined with a BCA assay kit (Pierce; Thermo Fisher Scientific, Inc.). Then each protein sample $(30 \mu \mathrm{g})$ was separated by $4-15 \%$ SDS-PAGE, followed by transfer onto nitrocellulose membranes (Amersham; GE Healthcare, Chicago, IL, USA). The membranes were blocked with 5\% non-fat milk in Tris buffer saline containing $0.1 \%$ Tween-20 (TBST) for $1 \mathrm{~h}$ at room temperature, and subsequently incubated overnight at $4^{\circ} \mathrm{C}$ with primary antibodies against GAPDH (cat. no. 5174; 1:1,000; Cell Signaling Technology, Inc.), E-cadherin (cat. no. 14472; 1:1,000; Cell Signaling Technology, Inc.), PDZD2 (cat. no. ab196631; 1:400; Abcam), PCNA (cat. no. 13110; 1:1,000; Cell Signaling Technology, Inc.), cleaved caspase-3 (cat. no. 9661; 1:1,000; Cell Signaling Technology, Inc.), and vimentin (cat. no. 5741; 1:1,000; Cell Signaling Technology, Inc.). After washing with TBST buffer, the protein bands were incubated with horseradish peroxidase (HRP)-conjugated secondary antibodies (cat. nos. ab205718 and ab205719; 1:20,000; Abcam) for $1 \mathrm{~h}$ at room temperature. Finally, the blots were visualized with ECL-Plus reagent (Millipore, Billerica, MA, USA). GAPDH was used as a loading control in the western blot analyses.

Immunofluorescence assays. Immunofluorescence assays were performed to estimate the levels of E-cadherin, vimentin, and PDZD2 in tumor cells. The cells were fixed with $4 \%$ formaldehyde in PBS for $15 \mathrm{~min}$ and then washed three times with PBS. Next, the cells were permeabilized with $100 \%$ methanol for $10 \mathrm{~min}$ at $-20^{\circ} \mathrm{C}$, blocked with $3 \%$ bovine serum albumin (BSA) in PBS for $60 \mathrm{~min}$, and then incubated overnight with primary antibodies anti-PDZD2 (cat. no. ab196631; 1:200; Abcam), anti-E-Cadherin (cat. no. 14472; 1:50; Cell Signaling 
Technology, Inc.) and anti-vimentin (cat. no. 5741; 1:100; Cell Signaling Technology, Inc.) at $4^{\circ} \mathrm{C}$. Following incubation, the cells were rinsed three times in PBS and placed onto coverslips that were incubated with a fluorochrome-conjugated secondary antibody for $1 \mathrm{~h}$ at room temperature in the dark. After staining the cell nuclei with DAPI, the coverslips were mounted onto glass slides with neutral gum and the cells were observed under an FV10i confocal microscope (Olympus Corporation, Tokyo, Japan).

Immunohistochemistry. Immunohistochemistry assays were performed to determine the expression of PDZD2 in tumor tissues as previously described (22). Formalin-fixed, paraffinembedded tissue sections (2- $\mu \mathrm{m}$ thickness) were prepared and subsequently incubated in xylene for $5 \mathrm{~min}, 100 \%$ ethanol for $10 \mathrm{~min}$, and then in $95 \%$ ethanol for $10 \mathrm{~min}$. Antigen unmasking was performed, and the slides were then blocked with $3 \%$ hydrogen peroxide for $30 \mathrm{~min}$ at room temperature. Next, the sections were incubated overnight at $4^{\circ} \mathrm{C}$ with a primary antibody against PDZD2, and then subsequently incubated with the secondary antibody. An EnVision Detection System kit (Dako, Glostrup, Denmark) was used to assess the immunostaining results.

Cell transfection. MG-63 cells were seeded into a 12-well plate and cultured to $\sim 60 \%$ confluence. The cells were then washed with PBS and transfected with miR-363 mimics $(50 \mathrm{nM})$, siRNA-PDZD2 $(1 \mu \mathrm{g} / \mathrm{ml})$ or the corresponding negative control for 24,48 and $72 \mathrm{~h}$, using $100 \mathrm{nM}$ Lipofectamine 2000 (Thermo Fisher Scientific, Inc.), according to the manufacturer's instructions. Following transfection for the indicated time period, the cells were harvested for use in further experiments.

Cell Counting Kit-8 (CCK-8) assay. MG-63 cells, transfected with the indicated agents, were harvested, washed with PBS, and then counted with a Cell Counting Kit-8 (Dojindo Molecular Technologies, Inc., Kumamoto, Japan). Other transfected cells were mixed with DMEM for use in cell viability assays. Absorbance was measured at $450 \mathrm{~nm}$ with a microplate reader.

$E d U$ assay. Cell proliferation was assessed by use of an EdU assay kit (Ruibo Biotechnology Co., Ltd., Guangzhou, China). Tumor cells $\left(1 \times 10^{5}\right.$ cells/well $)$ were incubated with $100 \mu$ l of $50 \mu \mathrm{M} \mathrm{EdU}$ per well for $2 \mathrm{~h}$ at $37^{\circ} \mathrm{C}$ in 24 -well plates, and then were fixed with $100 \mu \mathrm{l}$ of fixing buffer (4\% polyformaldehyde in PBS) for $30 \mathrm{~min}$ at room temperature. Subsequently, the cells were incubated for $5 \mathrm{~min}$ with $50 \mu \mathrm{l}$ of $2 \mathrm{mg} / \mathrm{ml}$ glycine, and washed with $100 \mu \mathrm{l}$ PBS. Next, the cells were treated with $0.5 \%$ Triton $\mathrm{X}$ and reacted with $1 \mathrm{X}$ Apollo solution for $30 \mathrm{~min}$. The cells were then incubated with $100 \mu$ l Hoechst 33342 solution for $30 \mathrm{~min}$ in the dark, and washed with $100 \mu \mathrm{l}$ PBS. Finally, the cells were analyzed by fluorescence microscopy (Lionheart; BioTek Instruments, Inc., Winooski, VT, USA).

Colony formation assay. MG-63 cells transfected with the indicated agents were harvested and then re-suspended in complete medium containing 10\% FBS. The re-suspended cells were then seeded into 12 -well plates $\left(1 \times 10^{3}\right.$ cells/well $)$ and treated as indicated for 10 days. Following fixation in methanol for $15 \mathrm{~min}$, the cells were stained with $0.1 \%$ crystal violet, and visualized under a dissection microscope (Olympus Corporation). Colonies consisting of $\geq 50$ cells were counted.

Apoptosis and cell cycle distribution analysis. Cell apoptosis was analyzed using the Hoechst 33258 assay, flow cytometry or the terminal deoxynucleotidyl-transferase-mediated dUTP nick end labelling (TUNEL) assay. For the Hoechst assay, MG-63 cells were harvested, washed with PBS, and then fixed with paraformaldehyde. The fixed cells were then stained with $0.1 \mu \mathrm{g} / \mathrm{ml}$ Hoechst 33258 solution (Sigma-Aldrich; Merck KGaA, Darmstadt, Germany), and changes in nuclear morphology were observed under a fluorescence microscope (Olympus Corporation). For flow cytometry, $2 \mu \mathrm{l}$ of Annexin V mixed with $2 \mu \mathrm{l}$ of propidium iodide (PI; eBioscience; Thermo Fisher Scientific, Inc.) were added to each tube of cells ( $1 \times 10^{5}$ cells per tube) for $30 \mathrm{~min}$, according to the manufacturer's instructions. The cell staining was then quantified by flow cytometry on a FACS Calibur instrument with CellQuest Pro v5.2 software (BD Biosciences, San Jose, CA, USA). For the TUNEL assay, tumor tissues were fixed in formalin, embedded in paraffin, and cut into $4-\mu \mathrm{m}$ sections. Then, tumor sections were stained using an apoptosis in situ detection kit (Wako Pure Chemical Industries, Ltd., Osaka, Japan), and according to the manufacturer's instructions. Five non-overlapping fields from each section were randomly selected and observed under a fluorescence microscope. To determine the cell cycle phase distribution, cells were stained with PI staining solution (10 $\mu \mathrm{g} /$ $\mathrm{ml} \mathrm{RNase} \mathrm{A;} 50 \mu \mathrm{g} / \mathrm{ml} \mathrm{PI}$ ) at $37^{\circ} \mathrm{C}$ for $30 \mathrm{~min}$ in the dark, and then analyzed using a flow cytometer equipped with Cell-Quest Pro v5.2 software (BD Biosciences).

Cell migration and invasion analysis. The scratch wound assay was used to determine the migration of MG-63 cells. Cells transfected with the indicated agents were allowed to form separate monolayers, which were then scratched by dragging a plastic tip across the monolayer's surface. Five fields were randomly selected, and the distances traveled by the migrated cells were measured under a light microscope. For the invasion assay, transwells coated with matrigel were used. A total of $2 \times 10^{4}$ MG-63 cells in serum-free media were added into the upper chambers of the matrigel-coated transwell inserts. DMEM with $10 \%$ FBS $(1 \mathrm{ml})$ was added into the bottom chambers. After $24 \mathrm{~h}$ incubation, cells that did not migrate or invade were removed using a cotton swab, stained with crystal violet, and then counted under an inverted microscope. The number of cells in each of five randomly selected microscopic fields was counted. Each experiment was repeated three times.

Xenograft animal model. Thirty male mice (4-5 weeks; 20-22 g; purchased from Tianjin Purcell Biotechnology Co., Ltd., Tianjin, China) were housed in a sterile room at $25^{\circ} \mathrm{C}$, with a 12-h light/dark cycle and free access to food and water. Human MG-63 cells $\left(2 \times 10^{6}\right)$ transfected with the lentivirus vector containing miR-363 mimics or the negative control were subcutaneously injected into the rear flank of each nude mouse (10 mice per group). The tumor sizes were measured at three-day intervals, and the tumor volumes were calculated as follows: $\mathrm{V}\left(\mathrm{cm}^{3}\right)=$ width ${ }^{2}\left(\mathrm{~cm}^{2}\right) \mathrm{x}$ length $(\mathrm{cm}) / 2$. The protocols for all animal studies were approved by the Institutional 

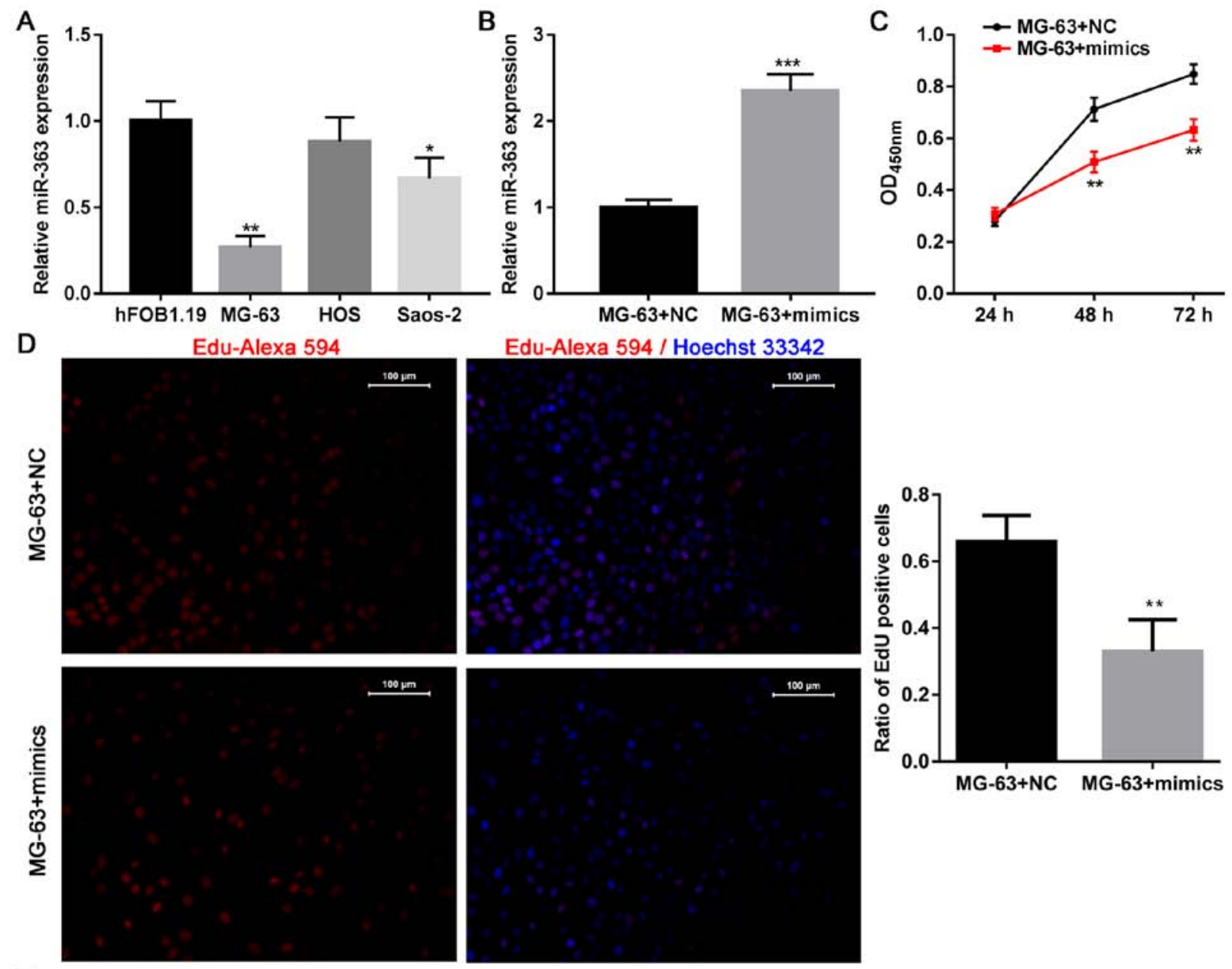

E
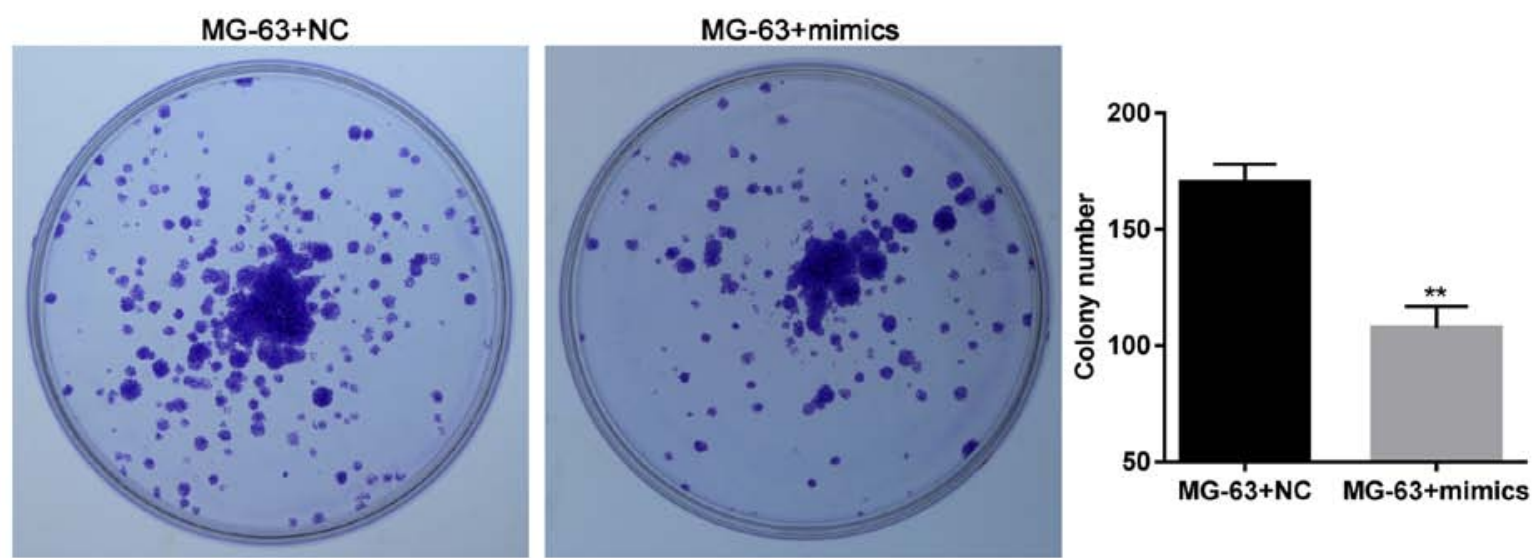

Figure 1. Restoration of miR-363 expression in OS cells inhibits cell proliferation in vitro. (A) Expression levels of miR-363 were determined in three OS cell lines (MG-63, HOS, and Saos2) and one human osteoblastic cell line (hFOB1.19) by reverse transcription-quantitative polymerase chain reaction. ${ }^{*} \mathrm{P}<0.05$ and ${ }^{* *} \mathrm{P}<0.01$ compared with hFOB1.19 cells. (B) miR-363 mimics and negative control were efficiently transfected into MG-63 cells. (C) Cell viability (CCK-8 assay), (D) proliferation (EdU assay) and (E) colony formation were evaluated in the cells transfected with either miR-363 mimics or negative control. Data are presented as mean \pm standard deviation. ${ }^{* *} \mathrm{P}<0.01$ and ${ }^{* * * *} \mathrm{P}<0.001$ compared with $\mathrm{NC}$ group. OS, osteosarcoma; NC negative control; OD, optical density.

Animal Care and Use Committee of Southern Medical University (Guangzhou, China).

Statistical analysis. Results are presented as the mean \pm standard deviation resulting from at least three independent repeats. Student's t-test or one-way (followed by Tukey's multiple comparisons test) or two-way (followed by Sidak's multiple comparisons test) analysis of variance was used to compare differences between two or more groups. All data were processed with SPSS 19.0 software (IBM Corp., Armonk, NY, USA). $\mathrm{P}<0.05$ was considered to indicate a statistically significant difference.

\section{Results}

Restoration of miR-363 in OS cells inhibits cell growth in vitro. Firstly, the role of miR-363 in OS was investigated in vitro. The levels of miR-363 expression were evaluated in three OS 
A

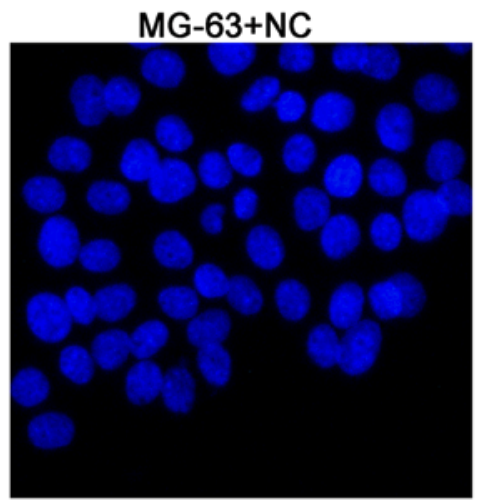

MG-63+mimics

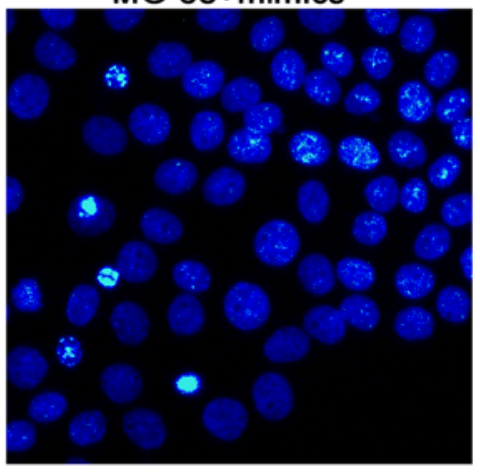

B
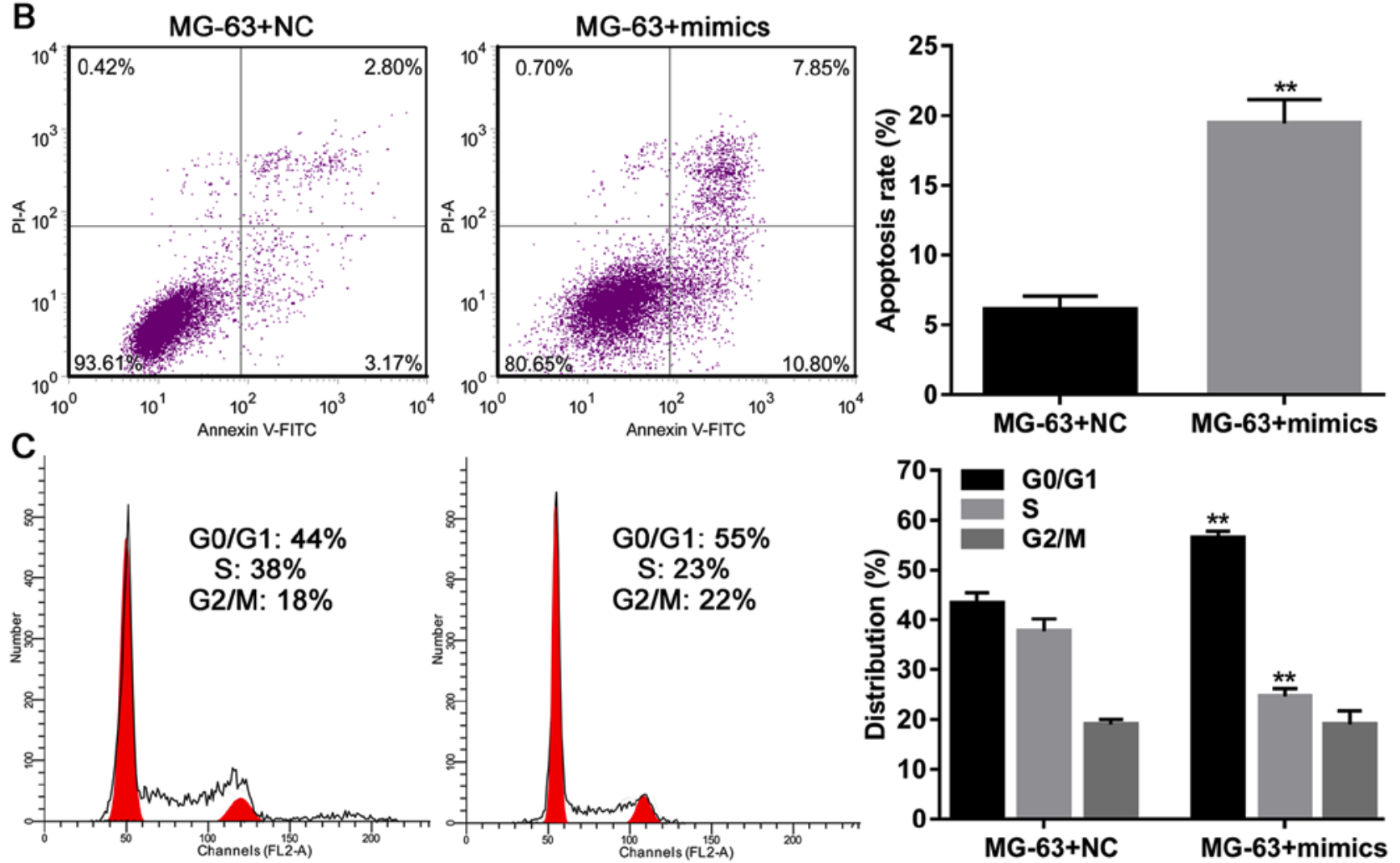

Figure 2. miR-363 overexpression in MG-63 cells induces apoptosis in vitro. (A) The apoptosis rate was determined by Hoechst staining (magnification, $\mathrm{x} 200$ ) and (B) flow cytometry. (C) Cell cycle distribution was determined by PI staining and flow cytometry analysis. Data are presented as mean \pm standard deviation. ${ }^{* *} \mathrm{P}<0.01$ compared with NC group. PI, propidium iodide; NC negative control; FITC, fluorescein isothiocyanate.

cell lines (MG-63, HOS and Saos2) and one human osteoblastic cell line (hFOB1.19). The present data demonstrated that the levels of miR-363 were lower in two out of three tumor cells lines tested, compared with the hFOB1.19 cells, and particularly in the MG-63 cells (Fig. 1A). Thus, overexpression of miR-363 was forced in MG-63 cells (Fig. 1B) and the subsequent effects were investigated on OS cell growth. The results demonstrated that overexpression of miR-363 significantly impaired the viability of MG-63 cells at 48 and $72 \mathrm{~h}$ (Fig. 1C). Overexpression of miR-363 significant impaired cell viability at 48 and $72 \mathrm{~h}$ in SaOS-2 cells as well (data not shown). In addition, the EdU assay indicated that the proliferation of MG-63 cells was impaired following transfection with miR-363 mimics (Fig. 1D), and colony formation assays revealed that cells transfected with the miR-363 mimics had a decreased ability to form colonies (Fig. 1E). Furthermore, Hoechst staining and flow cytometry analysis demonstrated that MG-63 cells overexpressing miR-363 had higher rates of apoptosis (Fig. 2A and B), and flow cytometry results demonstrated that miR-363 overexpression could induce G1/S arrest in MG-63 cells (Fig. 2C). In total, these findings indicated that miR-363 expression inhibited the growth and proliferation of OS cells by inducing apoptosis and G1/S arrest.

miR-363 overexpression impairs cell migration and invasion by regulating the EMT phenotype. Next, the effects of miR-363 overexpression on the migration and invasion abilities of MG-63 cells, and the potential underlying mechanisms, were investigated. Scratch wound assays demonstrated that MG-63 cell migration was significantly attenuated following miR-363 mimics transfection, compared with transfection with negative controls (Fig. 3A). Transwell invasion assays demonstrated that the invasion ability of MG-63 cells was also impaired following miR-363 overexpression (Fig. 3B). With regard to the 
A
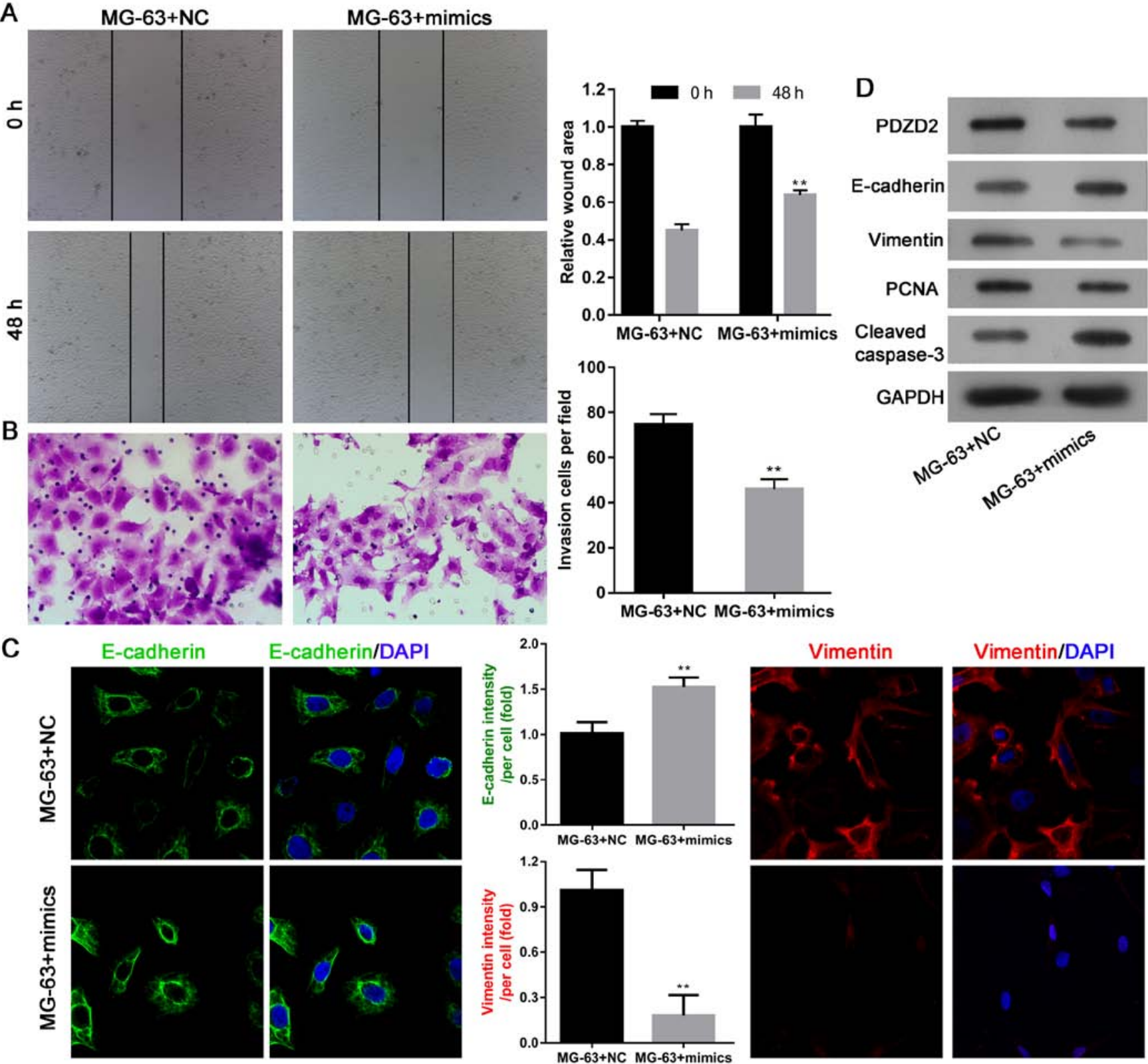

Figure 3. Restoration of miR-363 expression in osteosarcoma cells impairs their migration and invasion capabilities. (A) Migration of MG-63 cells was determined with the scratch wound assay. (B) Invasion of MG-63 cells was determined with the transwell assay (magnification, x200). (C) Expression of EMT markers E-cadherin and vimentin was evaluated by immunofluorescence (magnification, x200). (D) Western blot analysis of protein expression for the EMT markers as well as PZDZ2, PCNA, and cleaved caspase-3. Data are presented as mean \pm standard deviation. ${ }^{* *} \mathrm{P}<0.01$ compared with NC group. EMT, epithelial-mesenchymal transition; NC negative control; PZDZ2, PDZ domain containing 2; PCNA, proliferating cell nuclear antigen.

underlying mechanism, results from immunofluorescence and western blot analyses revealed that expression of the epithelial marker E-cadherin was upregulated following miR-363 overexpression, while the mesenchymal marker vimentin was downregulated (Fig. 3C and D). Furthermore, expression of the cell proliferation marker PCNA was decreased, while cleavage of caspase-3 was increased following miR-363 overexpression (Fig. 3D), suggesting that the tumor suppressive effect of miR-363 was dependent on PCNA, caspase-3, and the EMT phenotype.

PDZD2 is a direct target of miR-363 and promotes cell proliferation in vitro. The online prediction software miRWalk (http://www.umm.uni-heidelberg.de/apps/zmf/mirwalk/) was used to discover potential targets of miR-363, and the gene PDZD2 was identified. Further analysis revealed that the 3' untranslated region (UTR) of PDZD2 mRNA harbored a binding site for miR-363 (Fig. 4A); therefore, the interaction between PDZD2 and miR-363 was investigated in OS cells. The results demonstrated that overexpression of miR-363 in MG-63 cells significantly inhibited their ability to produce PDZD2 mRNA and protein (Fig. 4B and C). To confirm PDZD2 as a direct target of miR363, a luciferase reporter vector with the full-length 3 'UTR (wild-type or mutant) of PDZD2 was transfected into MG-63 cells for use in luciferase reporter assays. The results revealed that miR-363 mimics significantly impaired the luciferase activity of the wild-type PDZD2 3'UTR, but not the mutant control, indicating that 
A
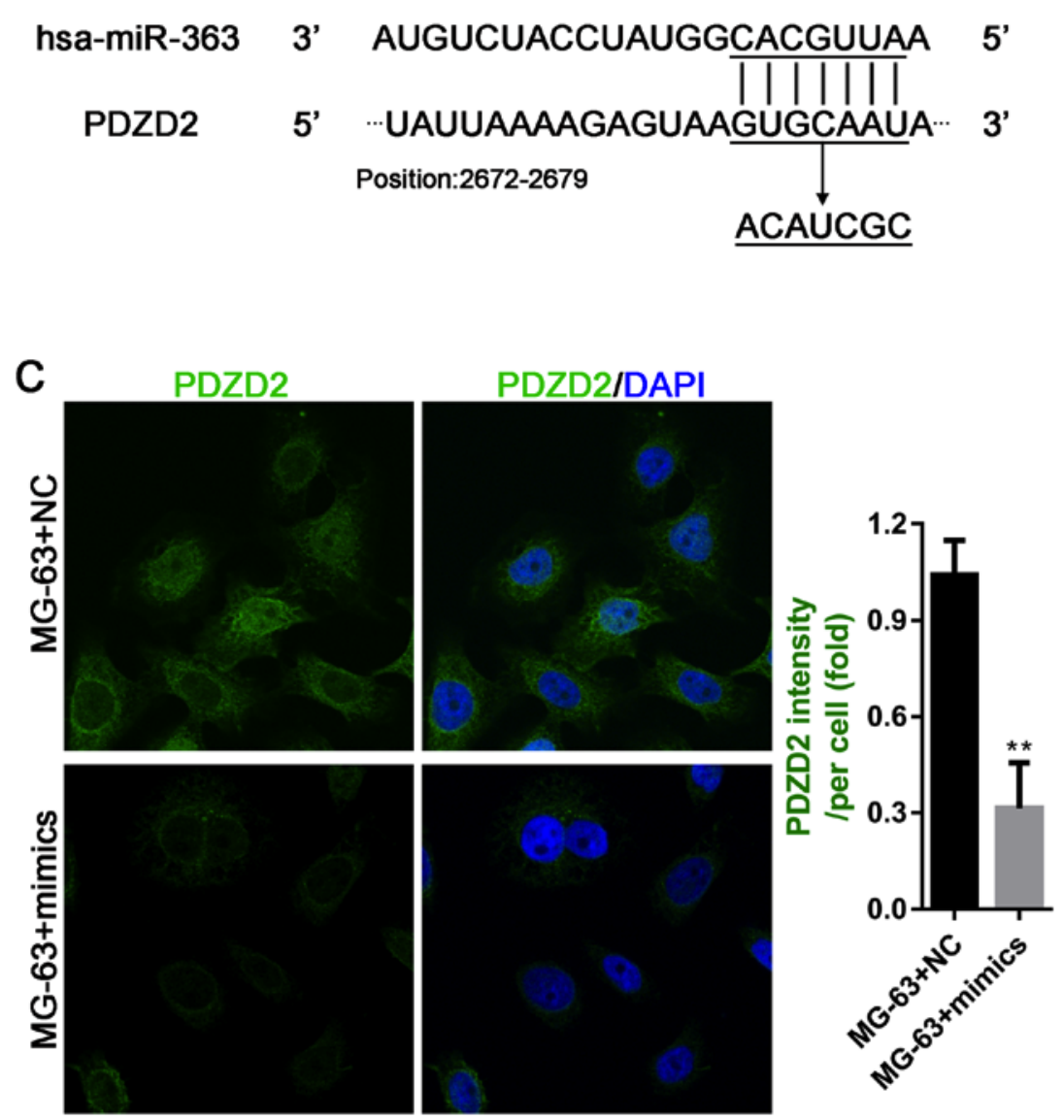

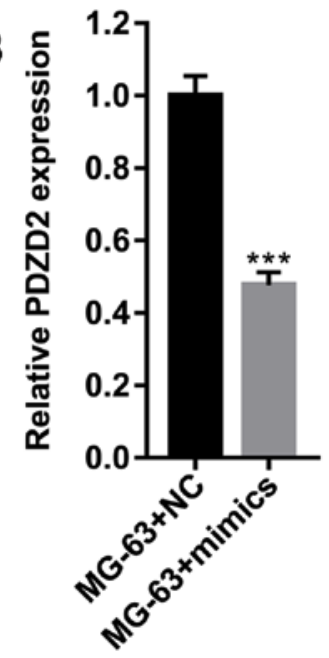

D

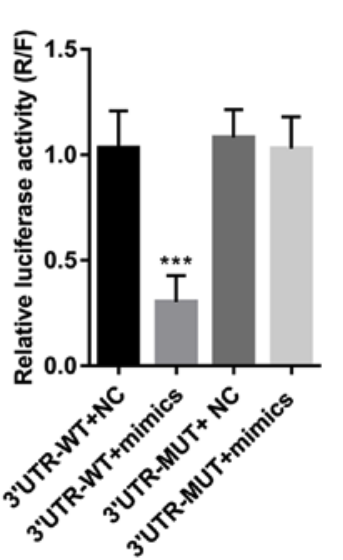

Figure 4. miR-363 directly targets PDZD2 in osteosarcoma cells. (A) The 3'UTR of PDZD2 mRNA harbors a binding site for miR-363 (underlined sequence). (B) Overexpression of miR-363 reduced the levels of PDZD2 mRNA and (C) protein in MG-63 cells, as determined by reverse transcription-quantitative polymerase chain reaction and immunofluorescence analysis (magnification, x200), respectively. (D) MG-63 cells were transfected with the full-length 3'UTR (wild-type or mutant) of PDZD2, and the luciferase reporter assay was used to confirm direct interaction. Data are presented as mean \pm standard deviation. ${ }^{* *} \mathrm{P}<0.01$ and ${ }^{* * *} \mathrm{P}<0.001$ compared with NC group. PZDZ2, PDZ domain containing 2; UTR; untranslated region; NC negative control; WT, wild-type; MUT, mutant.

miR-363 directly targeted PDZD2 in OS cells to decrease PDZD2 protein levels (Fig. 4D).

Next, the effects of decreased PDZD2 protein levels in OS cells were investigated. After efficiently knocking down PDZD2 expression in MG-63 cells by siRNA (Fig. 5A), the apoptosis (Fig. 5B), colony formation (Fig. 5C), and invasion capabilities of the cells were examined (Fig. 5D). The results demonstrated that knockdown of PDZD2 induced apoptosis and attenuated the colony formation and invasion capabilities of the cells. These effects were associated with a decrease in PCNA expression, increased cleavage of caspase- 3 , and reduced expression of the EMT phenotype (Fig. 5E). These findings suggested that PDZD2 could induce tumor suppression via miR363 in OS cells.

miR-363 expression restricts tumor growth with reduced PDZD2 levels in vivo. Because miR-363 and PDZD2 were demonstrated to regulate OS cells in vitro, we sought to determine the function of miR-363 in OS in vivo. MG-63 cells were transfected with miR-363 mimics or the negative control, and then injected into nude mice to produce a xenograft model of human MG-63 tumors. The results demonstrated that the mice injected with MG-63 cells overexpressing miR-363 developed significantly smaller tumors that displayed delayed growth (Fig. 6A and B). The successful upregulation of miR-363 (Fig. 6C) and downregulation of PDZD2 (Fig. 6D and E) was confirmed at the mRNA and protein levels in the tumor tissues. The tumor cell apoptosis rates were also estimated in the tumor tissues, and the results demonstrated that miR-363 markedly induced apoptosis of OS cells in vivo (Fig. 6F). These effects were associated with the decreased expression of PCNA, increased cleavage of caspase-3, and impairment of the EMT phenotype in the miR-363-overexpressing tumors compared with controls (Fig. 6G).

\section{Discussion}

Osteosarcoma is an aggressive malignant neoplasm that arises from primitive transformed cells of mesenchymal origin. The survival rate of patients with metastatic disease is $<30 \%$, and 


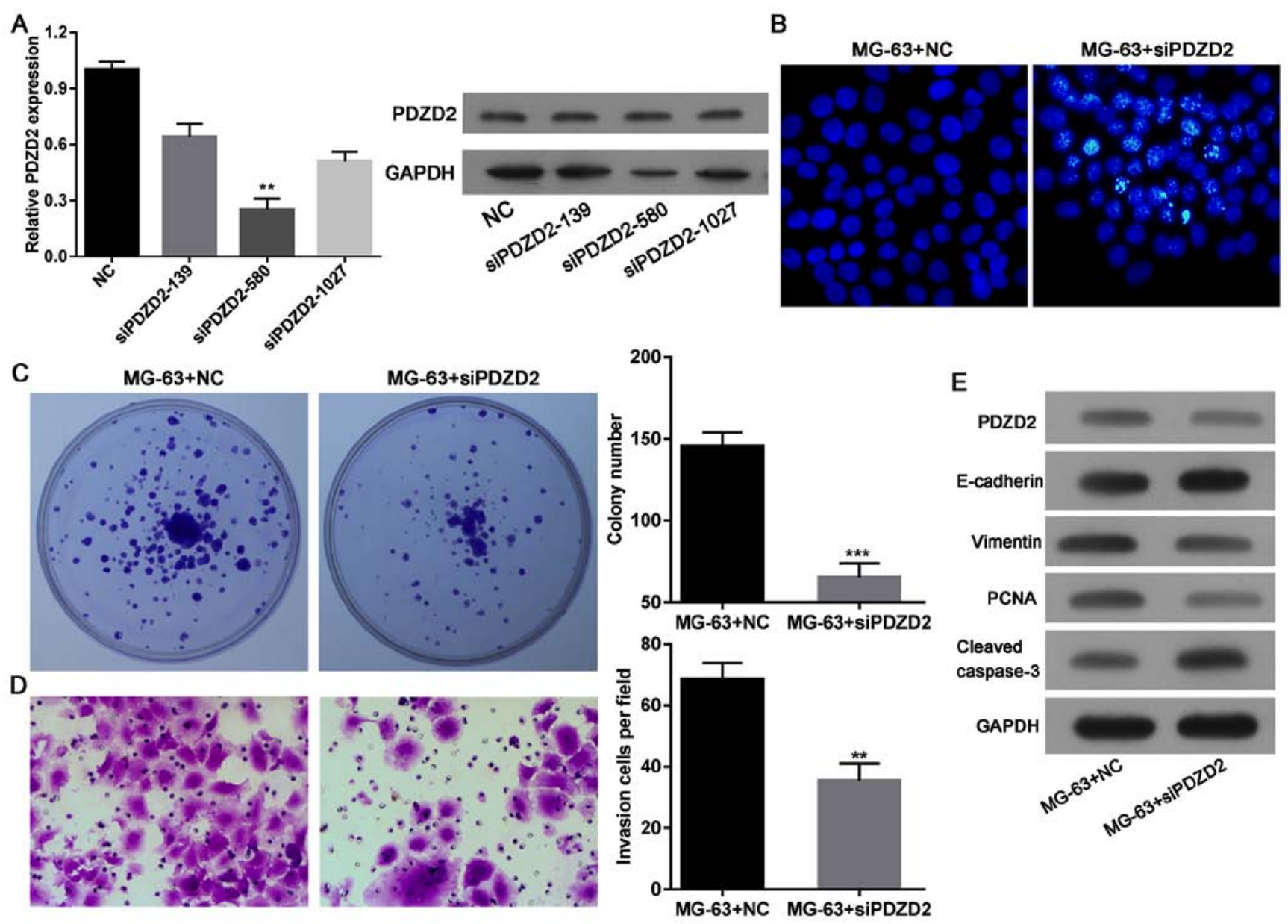

Figure 5. PDZD2 knockdown inhibits osteosarcoma cell growth and EMT. (A) Successful knockdown of PDZD2 was established by evaluating three separate siRNAs, and selecting the most efficient construct. (B) Cell apoptosis (magnification, $\mathrm{x} 200$ ), (C) colony formation and (D) invasion (magnification, $\mathrm{x} 200$ ) were evaluated in the MG-63 cells following PDZD2 knockdown. (E) Protein levels of PDZD2, EMT markers E-cadherin and vimentin, PCNA, and cleaved caspase-3 were determined by western blot analysis. Data are presented as mean \pm standard deviation. ${ }^{* *} \mathrm{P}<0.01$ and ${ }^{* * * *} \mathrm{P}<0.001$ compared with $\mathrm{NC}$ group. PZDZ2, PDZ domain containing 2; EMT, epithelial-mesenchymal transition; siRNA, small interfering RNA; PCNA, proliferating cell nuclear antigen; NC negative control.

further efforts, including novel treatment protocols, are needed to improve patient outcomes (23). Although recent studies have provided additional insights into both the oncogenic and tumor suppressive functions of miRNAs during the development of osteosarcoma, the exact molecular mechanisms underlying the miRNA/target interaction in OS remain to be fully identified (24). The in vitro and in vivo analyses included in the present study demonstrated that the tumor suppressor miR-363 was downregulated in OS cells and promoted tumor growth by directly targeting PDZD2. Although preliminary results from our group have indicated that the miR-363 levels are reduced in OS tissues when compared to normal tissues ( $n=8$, data not shown), further confirmation of these results is underway in our group with larger samples.

PDZD2 was originally thought to be an oncogene whose expression is upregulated in prostate tumor cell lines and human primary prostate tumors. The activation of PDZD2 expression is an early event in human prostate tumorigenesis (7). The present study demonstrated that PDZD2 expression was associated with OS tumor progression. A recent study of single nucleotide polymorphisms associated with renal cell carcinoma indicated that rs10054504 at PDZD2 was significantly associated with the risk for renal cell carcinoma in a Chinese population (22). However, the efficiency of therapies that target PDZD2 remains unclear. Human secreted PDZD2 (sPDZD2) was demonstrated to have tumor suppressive effects. sPDZD2 induced the senescence of prostate cancer cells via transcriptional activation of mutant or wild-type p53, and sensitized cancer cells to apoptosis induction via genotoxic stress (25). In addition, the antiproliferative effect of sPDZD2 in human cancer cells was demonstrated to be mediated by induction of $\mathrm{S}$ phase cell cycle arrest (26). The present study reported that inhibition of PDZD2 promoted the apoptosis of OS cells and abrogated the migration and invasion capabilities of OS cell lines. Furthermore, knockdown of PDZD2 significant reduced expression of the cell proliferation marker PCNA, induced the cleavage of caspase-3, and impaired the EMT phenotype in OS cells. Further studies will be required to fully elucidate that miR-363/PDZD2-related downstream signals and their functions in OS cells.

Dysregulation of miRNA during the development of cancer is a hallmark of malignant transformation. The tumor suppressor miR-363 belongs to the miR-92a family, which is a group of highly conserved miRNAs that include miR-25, 
A

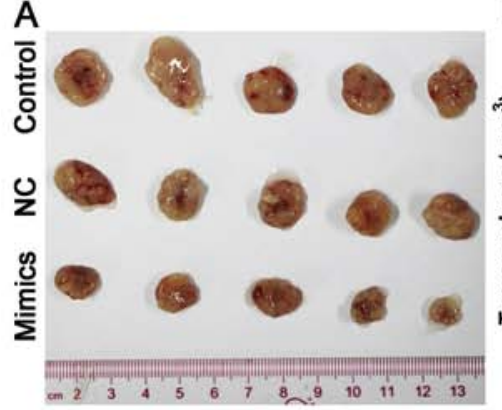

E

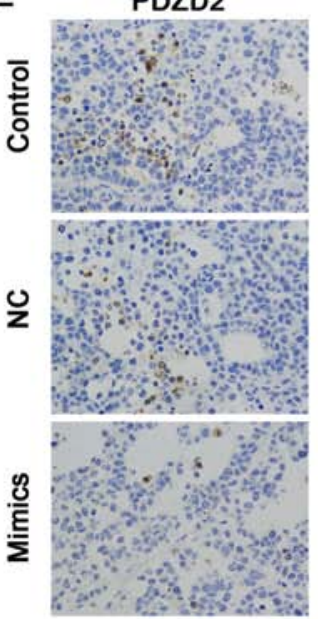

B

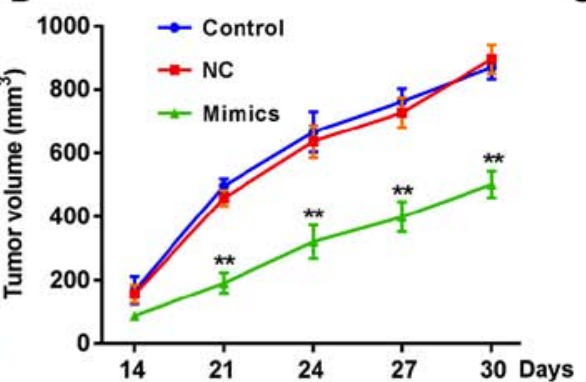

C

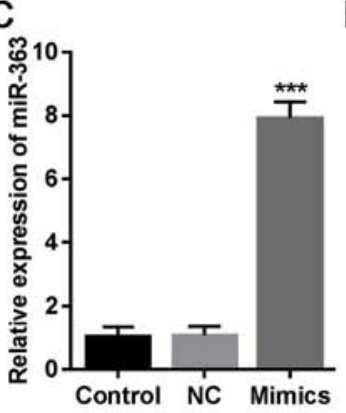

D

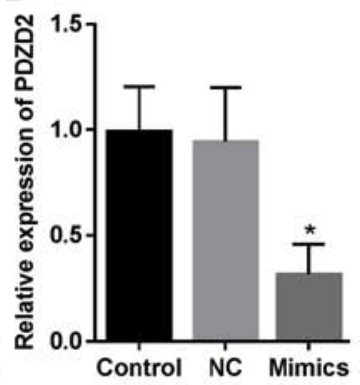

F FITC
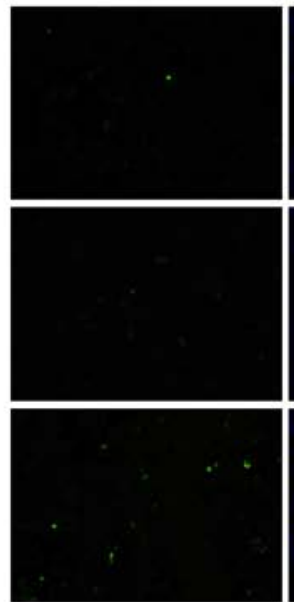
DAPI

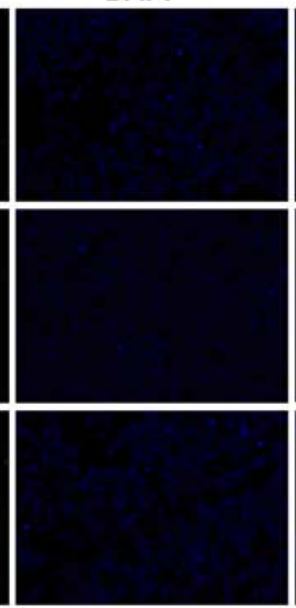

Merged

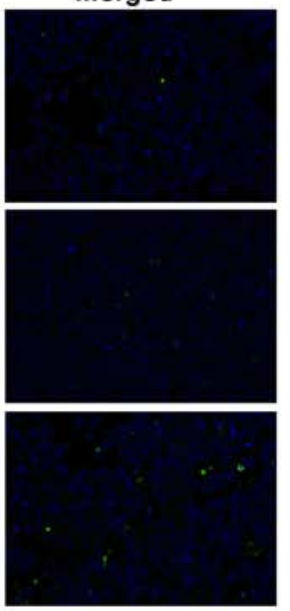

G

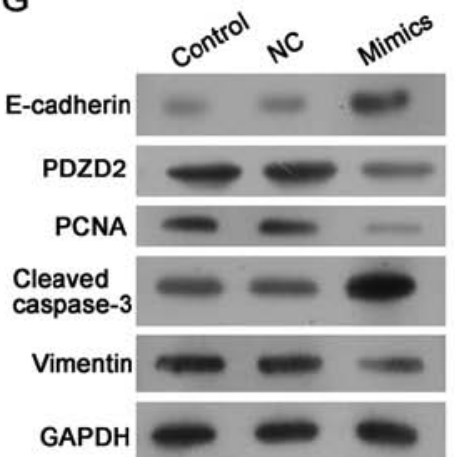

Figure 6. mir-363 induces the regression of osteosarcoma tumors in vivo. MG-63 cells ( 2x10 $)$, transfected with miR-363 mimics or negative control, were subcutaneously injected into the rear flanks of nude mice (5 mice per group). (A) Images of the tumors at the end of the experiment. (B) Quantification of the tumor volumes $\left(\mathrm{mm}^{3}\right)$ over time. (C) Expression levels of miR-363 and (D) PDZD2 mRNA in tumor tissues were analyzed by reverse transcription-quantitative polymerase chain reaction. (E) PDZD2 protein expression in tumor tissues was determined by immunohistochemistry (magnification, $\mathrm{x} 200$ ). (F) The apoptosis rates in the tumor sections were estimated by TUNEL staining (magnification, $x 200)$. (G) Protein expression levels of PDZD2, E-cadherin, vimentin, PCNA and cleaved caspase- 3 expression were determined by western blot analysis. Data are presented as mean \pm standard deviation. ${ }^{*} \mathrm{P}<0.05,{ }^{* *} \mathrm{P}<0.01$ and ${ }^{* * *} \mathrm{P}<0.001$ compared with NC group. PZDZ2, PDZ domain containing 2; PCNA, proliferating cell nuclear antigen; NC negative control.

miR-92a-1, miR-92a-2, and miR-363, and are associated with the formation of vascular endothelial cells (27). The aberrant expression of miR-92a family members has been reported in multiple types of cancer, and is related to carcinogenesis and tumor development (28). Zhou et al (29) found that the expression levels of miR-92a were significantly higher in colorectal cancer (CRC) tissues, and were correlated with an advanced clinical stage, lymph node metastasis and distant metastasis. miR-363-3p was demonstrated to be downregulated in CRC tissue specimens with lymph node metastasis, to promote CRC cell migration/invasion and induce EMT by increasing SRY-box 4 (Sox4) expression in vitro and in vivo (30). In prostate cancer, a high level of miR-363 in PC-3 cells promoted cell proliferation, induced cell transformation, and promoted EMT by targeting or inhibiting MYC proto-oncogene (31). The current study demonstrated that miR-363 levels in osteosarcoma cells were downregulated, which in turn resulted in upregulated expression of its target gene, PDZD2. Restoration of miR-363 expression in OS cells impaired cell viability, colony formation, migration and invasion, while it induced apoptosis and cell cycle arrest via PDZD2. These effects might be associated with decreases in PCNA, caspase-3, and the EMT phenotype. Similarly, Wang et al (32) demonstrated that miR-363 inhibited osteosarcoma cell proliferation and invasion by targeting SOX4. These findings indicate that miR-363 may function as a universal tumor suppressor in multiple types of cancer.

In conclusion, the present study reported PDZD2 as a novel target of the tumor suppressor miR-363 in OS cells. Overexpression of miR-363 or inhibition of PDZD2 promoted caspase-3-related apoptosis of tumor cells, inhibited EMT, and induced the regression of osteosarcoma tumors.

\section{Acknowledgements}

Not applicable.

\section{Funding}

No funding was received.

\section{Availability of data and materials}

All data generated or analyzed during this study are included in this published article.

\section{Author's contributions}

FH, LF and QY conceived and supervised the study, designed experiments, performed experiments, analyzed data, wrote the 
manuscript and made manuscript revisions. All authors read and approved the final manuscript.

\section{Ethics approval and consent to participate}

All protocols involving the use of animals were approved by the Institutional Animal Care and Use Committee of Southern Medical University (Guangzhou, China).

\section{Consent for publication}

Not applicable.

\section{Competing interests}

The authors declare that they have no competing interests.

\section{References}

1. Geller DS and Gorlick R: Osteosarcoma: A review of diagnosis, management, and treatment strategies. Clin Adv Hematol Oncol 8: 705-718, 2010.

2. Lamoureux F, Trichet V, Chipoy C, Blanchard F, Gouin F and Redini F: Recent advances in the management of osteosarcoma and forthcoming therapeutic strategies. Expert Rev Anticancer Ther 7: 169-181, 2007.

3. Gaspar N, Hawkins DS, Dirksen U, Lewis IJ, Ferrari S, Le Deley MC, Kovar H, Grimer R, Whelan J, Claude L, et al: Ewing sarcoma: Current management and future approaches through collaboration. J Clin Oncol 33: 3036-3046, 2015.

4. Misaghi A, Goldin A, Awad M and Kulidjian AA: Osteosarcoma: A comprehensive review. SICOT J 4: 12, 2018.

5. Thomas MK, Tsang SW, Yeung ML, Leung PS and Yao KM: The roles of the PDZ-containing proteins bridge-1 and PDZD2 in the regulation of insulin production and pancreatic beta-cell mass. Curr Protein Pept Sci 10: 30-36, 2009.

6. Ma RY, Tam TS, Suen AP, Yeung PM, Tsang SW, Chung SK, Thomas MK, Leung PS and Yao KM: Secreted PDZD2 exerts concentration-dependent effects on the proliferation of INS-1E cells. Int J Biochem Cell Biol 38: 1015-1022, 2006.

7. Chaib H, Rubin MA, Mucci NR, Li L, Day ML, Rhim JS and Macoska JA; Taylor JMG: Activated in prostate cancer: A PDZ domain-containing protein highly expressed in human primary prostate tumors. Cancer Res 61: 2390-2394, 2001

8. Yeung ML, Tam TS, Tsang AC and Yao KM: Proteolytic cleavage of PDZD2 generates a secreted peptide containing two PDZ domains. EMBO Rep 4: 412-418, 2003.

9. Zhang N, Wu Y, Gong J, Li K, Lin X, Chen H, Yu Y, Gou Y, Hou J, Jiang D, et al: Germline genetic variations in PDZD2 and ITPR2 genes are associated with clear cell renal cell carcinoma in Chinese population. Oncotarget 8: 24196-24201, 2017.

10. Panwar B, Arora A and Raghava GP: Prediction and classification of ncRNAs using structural information. BMC Genomics 15: 127, 2014

11. Bartel DP: MicroRNAs: Target recognition and regulatory functions. Cell 136: 215-233, 2009.

12. Shukla GC, Singh J and Barik S: MicroRNAs: Processing, maturation, target recognition and regulatory functions. Mol Cell Pharmacol 3: 83-92, 2011.

13. Hayes J, Peruzzi PP and Lawler S: MicroRNAs in cancer: Biomarkers, functions and therapy. Trends Mol Med 20: 460-469, 2014.

14. Di Leva G, Garofalo M and Croce CM: MicroRNAs in cancer. Annu Rev Pathol 9: 287-314, 2014.
15. Tian X, Zhang J, Yan L, Dong JM and Guo Q: MiRNA-15a inhibits proliferation, migration and invasion by targeting TNFAIP1 in human osteosarcoma cells. Int J Clin Exp Pathol 8: 6442-6449, 2015

16. Xu M, Jin H, Xu CX, Sun B, Mao Z, Bi WZ and Wang Y: miR-382 inhibits tumor growth and enhance chemosensitivity in osteosarcoma. Oncotarget 5: 9472-9483, 2014.

17. Tsuji S, Kawasaki Y, Furukawa S, Taniue K, Hayashi T, Okuno M, Hiyoshi M, Kitayama J and Akiyama T: The miR-363-GATA6Lgr5 pathway is critical for colorectal tumourigenesis. Nat Commun 5: 3150, 2014.

18. Zhou P, Huang G, Zhao Y, Zhong D, Xu Z, Zeng Y, Zhang Y, Li S and He F: MicroRNA-363-mediated downregulation of S1PR1 suppresses the proliferation of hepatocellular carcinoma cells. Cell Signal 26: 1347-1354, 2014.

19. Wang SH, Zhang WJ, Wu XC, Weng MZ, Zhang MD, Cai Q, Zhou D, Wang JD and Quan ZW: The lncRNA MALAT1 functions as a competing endogenous RNA to regulate MCL-1 expression by sponging miR-363-3p in gallbladder cancer. J Cell Mol Med 20: 2299-2308, 2016.

20. Zhang R, Li Y, Dong X, Peng L and Nie X: MiR-363 sensitizes cisplatin-induced apoptosis targeting in Mcl-1 in breast cancer. Med Oncol 31: 347, 2014

21. Livak KJ and Schmittgen TD: Analysis of relative gene expression data using real-time quantitative PCR and the 2(-Delta Delta C(T)) Method. Methods 25: 402-408, 2001.

22. Ding L, Ren J, Zhang D, Li Y, Huang X, Hu Q, Wang H, Song Y, Ni Y and Hou Y: A novel stromal lncRNA signature reprograms fibroblasts to promote the growth of oral squamous cell carcinoma via LncRNA-CAF/interleukin-33. Carcinogenesis 39: 397-406, 2018

23. Luetke A, Meyers PA, Lewis I and Juergens H: Osteosarcoma treatment - where do we stand? A state of the art review. Cancer Treat Rev 40: 523-532, 2014.

24. Liang W, Gao B, Fu P, Xu S, Qian Y and Fu Q: The miRNAs in the pathgenesis of osteosarcoma. Front Biosci 18: 788-794, 2013.

25. Tam CW, Cheng AS, Ma RY, Yao KM and Shiu SY: Inhibition of prostate cancer cell growth by human secreted PDZ domaincontaining protein 2 , a potential autocrine prostate tumor suppressor. Endocrinology 147: 5023-5033, 2006.

26. Tam CW, Liu VW, Leung WY, Yao KM and Shiu SY: The autocrine human secreted PDZ domain-containing protein 2 (sPDZD2) induces senescence or quiescence of prostate, breast and liver cancer cells via transcriptional activation of p53. Cancer Lett 271: 64-80, 2008

27. Li M, Guan X, Sun Y, Mi J, Shu X, Liu F and Li C: miR-92a family and their target genes in tumorigenesis and metastasis. Exp Cell Res 323: 1-6, 2014.

28. Olive V, Jiang I and He L: mir-17-92, a cluster of miRNAs in the midst of the cancer network. Int J Biochem Cell Biol 42. 1348-1354, 2010.

29. Zhou T, Zhang G, Liu Z, Xia S and Tian H: Overexpression of miR-92a correlates with tumor metastasis and poor prognosis in patients with colorectal cancer. Int J Colorectal Dis 28: 19-24, 2013.

30. Hu F, Min J, Cao X, Liu L, Ge Z, Hu J and Li X: MiR-363-3p inhibits the epithelial-to-mesenchymal transition and suppresses metastasis in colorectal cancer by targeting Sox4. Biochem Biophys Res Commun 474: 35-42, 2016.

31. Chen Y, Lu X, Wu B, Su Y, Li J and Wang H: MicroRNA 363 mediated positive regulation of c-myc translation affect prostate cancer development and progress. Neoplasma 62: 191-198, 2015.

32. Wang K, Yan L and Lu F: miR-363-3p Inhibits Osteosarcoma Cell Proliferation and Invasion via Targeting SOX4. Oncol Res 27: $157-163,2019$.

This work is licensed under a Creative Commons Attribution-NonCommercial-NoDerivatives 4.0 International (CC BY-NC-ND 4.0) License. 